\title{
Correction to: Basic mechanisms of escape of a harmonically forced classical particle from a potential well
}

\author{
O. V. Gendelman $(\mathbb{D}$ - G. Karmi
}

Published online: 9 December 2019

(C) Springer Nature B.V. 2019

\section{Correction to:}

\section{Nonlinear Dyn}

https://doi.org/10.1007/s11071-019-04985-9

Abstract This corrigendum corrects Eqs. (15-16) in the original paper. Other results, conclusions and discussion are not affected by this correction.

Quantitative evaluations presented in paper [1] in formulas (15-16) were evaluated for sets of parameters and initial conditions different from those mentioned in the paper. Correct results for zero initial conditions are presented below:

$$
\begin{aligned}
& F_{t r}(3,0)=F_{c}(3,0)=2 ; \\
& F_{t r}(1 / 3,0)=F_{c}(1 / 3,0)=2 / 3 \\
& F_{t r}(2,0)=\frac{2}{\sqrt{3}} \approx 1.15>F_{c}(2,0) \\
& F_{t r}(1 / 2,0)=\frac{1}{\sqrt{3}} \approx 0.577>F_{c}(1 / 2,0)
\end{aligned}
$$

The original article can be found online at https://doi.org/10. 1007/s11071-019-04985-9.

O. V. Gendelman $(\varangle) \cdot$ G. Karmi Faculty of Mechanical Engineering, Technion - Israel Institute of Technology, 3200003 Haifa, Israel e-mail: ovgend@tx.technion.ac.il

$$
\begin{aligned}
& F_{t r}(3, \pi / 2)=\frac{432}{25 \sqrt{11}} \approx 5.21>F_{c}(3, \pi / 2) ; \\
& F_{t r}(1 / 3, \pi / 2)=\frac{48}{25 \sqrt{11}} \approx 0.579>F_{c}(1 / 3, \pi / 2) ; \\
& F_{t r}(2, \pi / 2)=F_{c}(2, \pi / 2)=3 / 2 ; \\
& F_{t r}(1 / 2, \pi / 2)=F_{c}(1 / 2, \pi / 2)=3 / 8 ;
\end{aligned}
$$

Neither other results nor conclusions in the paper are affected by this correction. We are very grateful to Ms. Esther Goldberger who attracted our attention to the inconsistency, and apologize for the inconvenience.

Compliance with ethical standards

Conflict of interests The authors declare that they have no conflict of interests

\section{Reference}

1. Gendelman, O.V., Karmi, G.: Basic mechanisms of escape of a harmonically forced classical particle from a potential well. Nonlinear Dyn. https://doi.org/10.1007/ s11071-019-04985-9

Publisher's Note Springer Nature remains neutral with regard to jurisdictional claims in published maps and institutional affiliations. 